Case Report

\title{
A Case of Advanced Descending Colon Cancer in an Adult Patient with Intestinal Malrotation
}

\author{
Yoshifumi Nakayama, ${ }^{1,2}$ Masaki Akiyama, ${ }^{1,2}$ Yusuke Sawatsubashi, ${ }^{1,2}$ Noritaka Minagawa, \\ Takayuki Torigoe, ${ }^{1}$ and Keiji Hirata ${ }^{1}$ \\ ${ }^{1}$ Department of Surgery 1, School of Medicine, University of Occupational and Environmental Health, 1-1 Iseigaoka, \\ Yahata-nishi-ku, Kitakyushu 807-8555, Japan \\ ${ }^{2}$ Department of Gastroenterological and General Surgery, Wakamatsu Hospital of University of Occupational and \\ Environmental Health, 1-17-1 Hamamachi, Wakamatsu-ku, Kitakyushu 808-0024, Japan
}

Correspondence should be addressed to Yoshifumi Nakayama; nakayama@med.uoeh-u.ac.jp

Received 26 October 2015; Accepted 14 February 2016

Academic Editor: Daniel C. Damin

Copyright (C) 2016 Yoshifumi Nakayama et al. This is an open access article distributed under the Creative Commons Attribution License, which permits unrestricted use, distribution, and reproduction in any medium, provided the original work is properly cited.

This report presents an operative case of advanced descending colon cancer in an adult patient with intestinal malrotation. A 63-year-old Japanese male was suffering from left side abdominal pain, abdominal distension, and constipation. An endoscopic examination revealed an advanced tumor in the descending colon. Computed tomography (CT) of the abdomen revealed the thickening of the descending colon wall and superior mesenteric vein rotation. An opaque enema detected severe stenosis of the descending colon. An abdominal X-ray examination revealed the dilation of the colon and small intestine with niveau. At the insertion of an ileus tube, the C-loop of the duodenum was observed to be absent and the small intestine was located on the right side of the abdomen. After the decompression of the bowel contents, laparotomy was performed. Descending colon cancer was observed to have directly invaded the left side of the transverse colon. Left hemicolectomy, lymph node dissection, and appendectomy were performed. The patient had an uneventful recovery and was discharged from the hospital on the 16th day after surgery. This report presents a rare operative case of descending colon cancer in an adult patient with intestinal malrotation.

\section{Introduction}

Intestinal malrotation can be defined as any deviation from the normal 270-degree rotation of the midgut in a counterclockwise direction during embryonic development [1]. Malrotation or nonrotation of the midgut occurs in approximately 1 in 500 live births [1]. Intestinal malrotation is diagnosed during the first month of life in $85-90 \%$ of all cases [2]. Intestinal malrotation is rarely present in adulthood. A barium enema study of 2000 adults demonstrated that the prevalence of malrotation was $0.2 \%$ [3]. Synchronous colon cancer in adult patients with intestinal malrotation is extremely rare [4].

This report presents a rare case of descending colon cancer in an adult patient with intestinal malrotation.

\section{Case Report}

A 63-year-old Japanese male presented with the chief complaints of left side abdominal pain, abdominal distension, and constipation. As the patient's symptoms worsened, he underwent an endoscopic examination, which revealed the presence of an ulcerated tumor of the descending colon. $\mathrm{He}$ was referred to the surgical outpatient clinic of our hospital with advanced descending colon cancer.

The laboratory data showed the patient had a white blood cell count of $6,200 / \mathrm{mm}^{3}$, a hemoglobin level of $8.7 \mathrm{~g} / \mathrm{dL}$, a hematocrit level of $25.3 \%$, and a platelet count of $401,000 / \mathrm{mm}^{3}$. The patient's electrolyte and blood urea nitrogen levels were normal, as was his liver function. His CEA and CA19-9 levels were $1.7 \mathrm{ng} / \mathrm{mL}$ and CA19-9 was $21.3 \mathrm{U} / \mathrm{mL}$, 


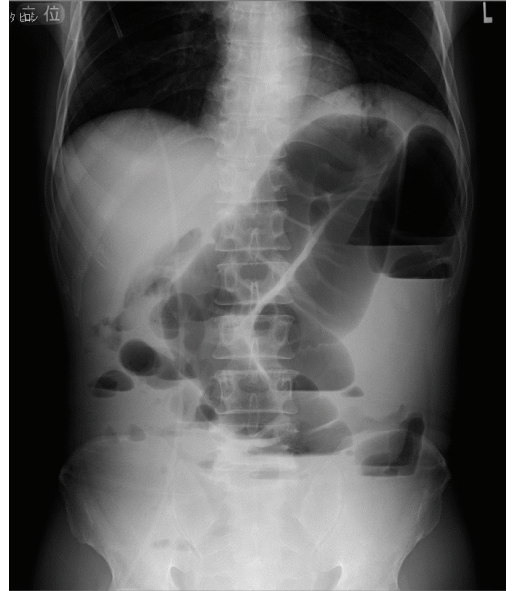

(a)

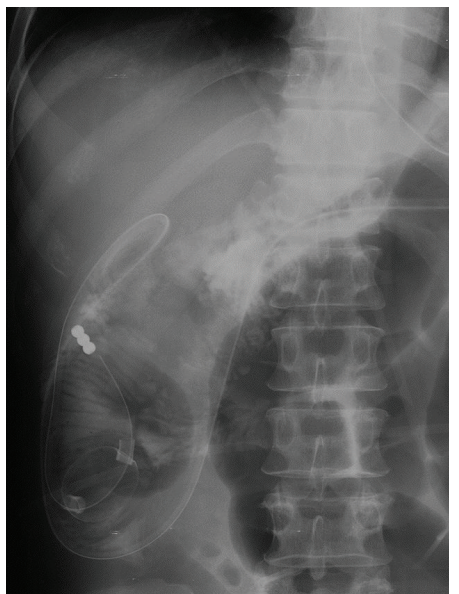

(b)

Figure 1: An abdominal X-ray examination revealed the dilation of the colon and niveau (a). At the insertion of an ileus tube, the C-loop of the duodenum was observed to be absent and the small intestine was located on the right side of the abdomen (b).

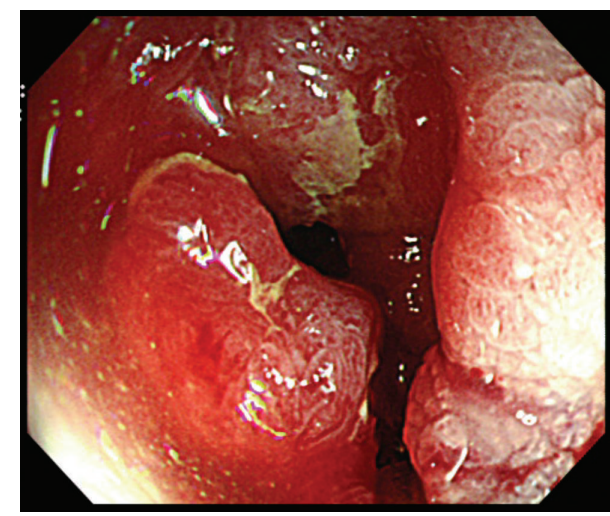

(a)

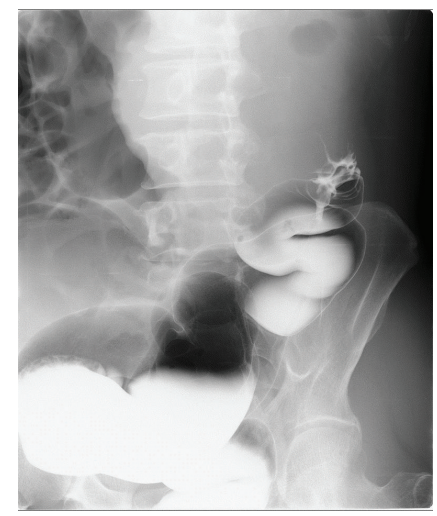

(b)

FIGURE 2: Colonoscopy revealed an advanced tumor in the descending colon (a). An opaque enema using contrast fluid revealed severe stenosis of the descending colon. The contrast fluid could not pass the stenotic part of the descending colon (b).

respectively. An abdominal X-ray examination revealed a dilated colon and niveau (Figure 1(a)). These findings were consistent with bowel obstruction. Computed tomography (CT) of the abdomen revealed the thickening of the descending colon wall, which was indicative of advanced descending colon cancer (Figure 3(a)); superior mesenteric vein rotation was also observed (Figure 3(b)). Colonoscopy revealed an advanced tumor in the descending colon (Figure 2(a)). The biopsy specimen was found to be moderately differentiated tubular adenocarcinoma. An opaque enema, using contrast fluid, revealed severe stenosis of the descending colon. The contrast fluid could not pass the stenosis (Figure 2(b)). At the insertion of an ileus tube, the C-loop of the duodenum was observed to be absent and the small intestine was located on the right side of the abdomen (Figure 1(b)). After the decompression of the bowel contents, laparotomy was performed. The small intestine was located on the right side of the abdomen. The appendix, cecum, and ascending colon were located in the middle abdomen. Descending colon cancer was observed to have directly invaded the left side of the transverse colon (Figure 4(a)). Left hemicolectomy, lymph node dissection, and appendectomy were performed. A histopathological examination with hematoxylin and eosin staining of the resected lesion revealed moderately differentiated adenocarcinoma (Figure 4(b)). No metastatic regional lymph nodes were identified. However, some lymphatic and venous invasion of the carcinoma cells was observed.

The patient had an uneventful recovery and was discharged from the hospital on the 16th day after surgery. He has been followed up in the outpatient clinic without recurrence for approximately 9 years.

\section{Discussion}

Given that intestinal malrotation in adults is not common and is usually asymptomatic [3], the clinical importance of intestinal malrotation in adults is less well documented than in children. Most of the clinical symptoms of this disorder are 


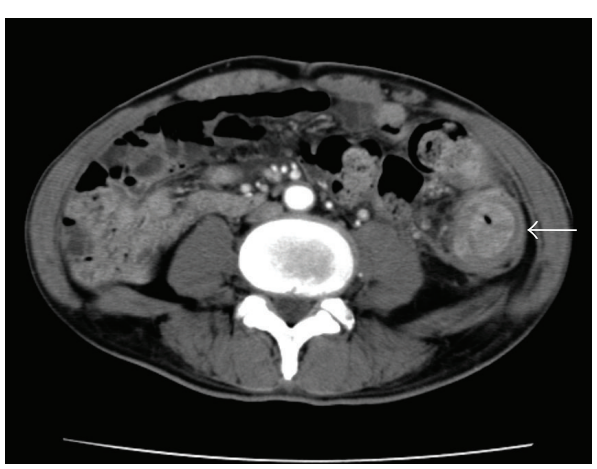

(a)

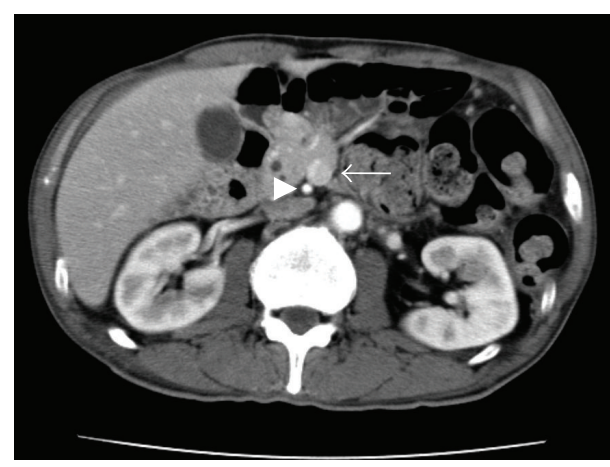

(b)

FIGURE 3: Computed tomography (CT) of the abdomen revealed thickening of the descending colon wall, which was indicative of advanced descending colon cancer (arrow) (a); superior mesenteric vein rotation was also observed (arrow) (b).

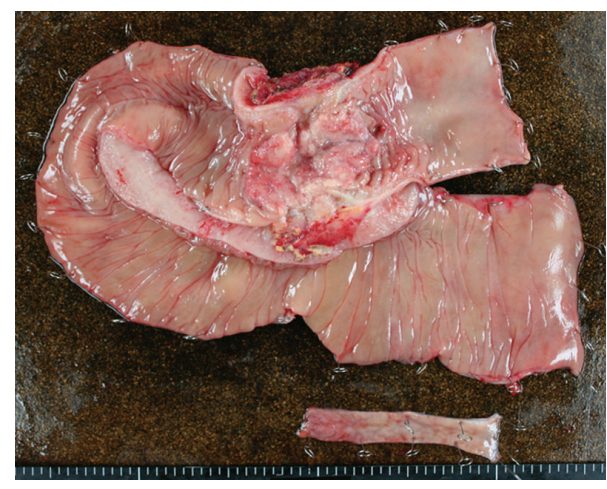

(a)

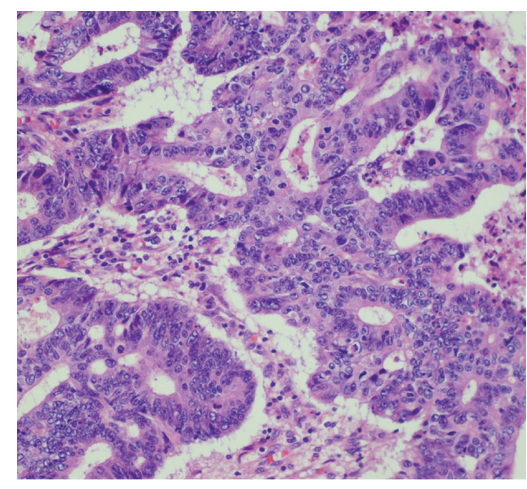

(b)

FIGURE 4: An operative specimen revealed advanced descending colon cancer with direct invasion of the transverse colon (a). A histopathological examination with hematoxylin and eosin staining confirmed that the lesion was moderately differentiated tubular adenocarcinoma (b).

due to the presence of coincidental intestinal disease. Many cases of adult intestinal malrotation are only discovered coincidentally at surgery for other diseases. In the present case, clinical symptoms of the patient, which included abdominal pain, abdominal distension, and constipation, occurred due to descending colon cancer with bowel obstruction, which was coincidental with the intestinal malrotation.

Intestinal malrotation has been divided into four types: nonrotation type, malrotation type, reversed rotation type, and paraduodenal hernia type, according to the arrest point of development (during embryonic development) anywhere along the rotation of the midgut in a counterclockwise direction [17]. Kato et al. summarized the Japanese literature and reported the frequency of each of the types of adult intestinal malrotation (nonrotation type (53.3\%), malrotation type (31.1\%), reversed rotation type (6.7\%), and paraduodenal hernia type (8.9\%)) [18]. The present case seemed to correspond to the nonrotation type, which is the most frequent one. Moreover, in $82.8 \%$ of the colorectal cancer patients with intestinal malrotation, the malrotation was classified as nonrotation type [19]. Table 1 shows that nonrotation type malrotation occurred most frequently $(75.0 \%)$ in colorectal cancer patients with intestinal malrotation.
Table 1 summarized the cases of colon cancer in adult patients with intestinal malrotation that are reported in the English literature, excepting the coexistent cases with situs inversus totalis [4-16]. Most of the patients had right-sided colon cancer (Table 1). Left-sided colon cancer is rare in adult patients with intestinal malrotation. The rate of leftsided colon cancer was $14.3 \%$ (Table 1). Anatomical disorders of the placement in this type of intestinal malrotation may be associated with carcinogenesis in the colon. Ren and $\mathrm{Lu}$ suggested that malrotation of the gut which may cause chronic bowel obstruction may lead to inflammation and carcinogenesis [8].

There are some problems at the operation in the cases of synchronous colon cancer in adult patients with intestinal malrotation. One of these problems is arterial and venous variations. Before the operation, confirm the presence of arterial and venous variations via enhanced CT. In the case of our patient, superior mesenteric vein rotation (Figure 3(b)) and anomalies were identified. It is important to understand vessel anomalies on CT angiography or enhanced CT scan before surgery [19]. Based on the imaging information, the regional lymph node dissection could be performed along with the arteries. 
TABLE 1: Case reports of colon cancer in adult patient with intestinal malrotation in English literature, which except the coexistent cases with situs inversus totalis.

\begin{tabular}{|c|c|c|c|c|c|c|}
\hline Number & Citation including year & Age & Sex & Location & Histologic type & Type of IM \\
\hline 1 & Gilbert et al., 1990 [5] & 55 & $\mathrm{M}$ & Splenic flexure & Adenoca. & Nonrotation \\
\hline 2 & Torreggiani et al., 2001 [6] & 86 & $\mathrm{~F}$ & Cecum & Carcinoma & Nonrotation \\
\hline 3 & Uchida et al., 2004 [7] & 57 & M & Transverse colon & & Nonrotation \\
\hline 4 & Ren and Lu, 2009 [8] & 45 & M & Ascending colon & Well to mod. diff. & Nonrotation \\
\hline 5 & Brillantino et al., 2009 [9] & 34 & M & Cecum & Well diff. & Nonrotation \\
\hline 6 & Michalopoulos et al., 2010 [10] & 76 & M & Ascending colon & Neoplasm & Reversed rotation \\
\hline 7 & Morimoto et al., 2012 [11] & 57 & M & Cecum & Cancer & Reversed rotation \\
\hline 8 & Donaire et al., 2013 [12] & 52 & M & Right colon & Adenoca. & Nonrotation \\
\hline 9 & Hirano et al., 2013 [13] & 68 & $\mathrm{~F}$ & Ascending colon & Well diff. & Nonrotation \\
\hline 10 & Hirano et al., 2013 [14] & 82 & $\mathrm{~F}$ & Transverse colon & Well diff. & Reversed rotation \\
\hline 11 & Enomoto et al., 2014 [15] & 48 & M & Transverse colon & Well to mod. diff. & Nonrotation \\
\hline 12 & $\mathrm{Lu}, 2014[4]$ & 43 & $\mathrm{~F}$ & Cecum & Adenosquamous ca. & NA \\
\hline 13 & Ray et al., 2014 [16] & 60 & $\mathrm{~F}$ & Ascending colon & Mod. diff. & NA \\
\hline 14 & Our case, 2015 & 63 & M & Descending colon & Mod. diff. & Nonrotation \\
\hline
\end{tabular}

IM: intestinal malrotation. NA: no assessment.

We herein presented a rare operative case of descending colon cancer in a patient with intestinal malrotation.

\section{Consent}

Written informed consent was obtained from the patient for publication of this case report.

\section{Conflict of Interests}

Yoshifumi Nakayama and the other coauthors have no conflict of interests to declare.

\section{Authors' Contribution}

Yoshifumi Nakayama contributed to the drafting and the editing of the paper. Masaki Akiyama and Yusuke Sawatsubashi contributed to obtaining the clinical details. Noritaka Minagawa contributed to literature search. Takayuki Torigoe helped in drafting the paper. Keiji Hirata helped in editing of the paper.

\section{References}

[1] A. M. Torres and M. M. Ziegler, "Malrotation of the intestine," World Journal of Surgery, vol. 17, no. 3, pp. 326-331, 1993.

[2] R. McIntosh and E. J. Donovan, "Disturbances of rotation of the intestinal tract: clinical picture based on observations in twenty cases," The American Journal of Disease of Children, vol. 57, no. 1, pp. 116-166, 1939.

[3] JL. Kantor, "Anomalies of the colon," Radiology, vol. 23, no. 6, pp. 651-662, 1934.

[4] I. L. Lu, "Adenosquamous carcinoma of the cecum with malrotation and intussusceptions," Journal of Cancer Research and Practice, vol. 30, no. 1, pp. 70-74, 2014.

[5] H. W. Gilbert, M. H. Thompson, and C. P. Armstrong, "The presentation of malrotation of the intestine in adults," Annals of the Royal College of Surgeons of England, vol. 72, no. 4, pp. 239-242, 1990.
[6] W. C. Torreggiani, F. Thornton, L. Lyburn, C. Brenner, and M. J. Lee, "Malrotation of the bowel resulting in a left-sided caecal carcinoma presenting as a palpable intrahernial mass," Australasian Radiology, vol. 45, no. 3, pp. 362-364, 2001.

[7] H. Uchida, Y. J. Kawamura, K. Takegami et al., "Colon cancer complicated by vascular and intestinal anomaly," HepatoGastroenterology, vol. 51, no. 55, pp. 156-158, 2004.

[8] P.-T. Ren and B.-C. Lu, "Intestinal malrotation associated with colon cancer in an adult: report of a case," Surgery Today, vol. 39, no. 7, pp. 624-627, 2009.

[9] A. Brillantino, L. Marano, M. Schettino et al., "Report of a rare case of colon cancer complicated by anomalies of intestinal rotation and fixation: a case report," Cases Journal, vol. 2, no. 9, article 6555, 2009.

[10] A. Michalopoulos, V. Papadopoulos, D. Paramythiotis et al., "Colonic cancer in a patient with intestinal malrotation: a case report," Techniques in Coloproctology, vol. 14, supplement 1, pp. S65-S66, 2010.

[11] M. Morimoto, H. Horie, H. Kumano, A. Lefor, K. Utano, and Y. Yasuda, "Reversed intestinal malrotation with concurrent cecal carcinoma," Asian Journal of Endoscopic Surgery, vol. 5, no. 3, pp. 149-151, 2012.

[12] M. Donaire, J. Mariadason, D. Stephens, S. Pillarisetty, and M. K. Wallack, "Carcinoma of the colon in an adult with intestinal malrotation," Case Reports in Surgery, vol. 2013, Article ID 525081, 4 pages, 2013.

[13] Y. Hirano, M. Hattori, D. Yagi, K. Maeda, K. Douden, and Y. Hashizume, "Laparoscopic surgery for the ascending colon cancer associated with malrotation of the midgut," Indian Journal of Surgery, vol. 75, no. 1, supplement, pp. 71-73, 2013.

[14] Y. Hirano, M. Hattori, M. Fujita, Y. Nishida, K. Douden, and Y. Hashizume, "The transverse colon cancer with the reversed rotation of the midgut treated with single incision laparoscopic colectomy," Indian Journal of Surgery, vol. 75, supplement 1, pp. S195-S198, 2013.

[15] H. Enomoto, H. Kawahara, M. Tomoda, K. Watanabe, T. Akiba, and K. Yanaga, "Laparoscopic surgery for the transverse colon carcinoma associated with non-rotarion type intestinal 
malrotation," Jikeikai Medical Journal, vol. 61, no. 4, pp. 87-92, 2014.

[16] D. Ray, C. Chattopadhyay, and S. Das, "Malrotation of intestine with carcinoma colon in adult," Journal of Nepal Medical Association, vol. 52, no. 8, pp. 727-729, 2014.

[17] C. A. Wang and C. E. Welch, "Anomalies of intestinal rotation in adolescents and adult," Surgery, vol. 54, pp. 839-855, 1963.

[18] K. Kato, H. Sakurai, S. Matsuda, and H. Suzuki, "A case of acute appendicitis presenting with left lower abdominal pain associated with malrotation of the intestine," The Journal of the Japanese Practical Surgeon Society, vol. 57, no. 10, pp. 2494-2498, 1996 (Japanese).

[19] C. Maeda, E. Hidaka, T. Uchida, T. Omoto, F. Ishida, and S. Kudo, "A case of transverse colon cancer with adult intestinal malrotation," Journal of Japan Surgical Association, vol. 74, no. 11, pp. 3103-3107, 2013 (Japanese). 


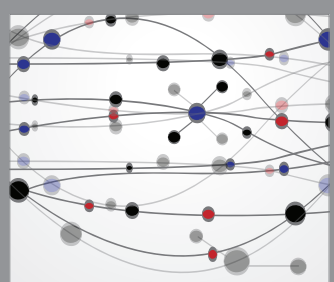

The Scientific World Journal
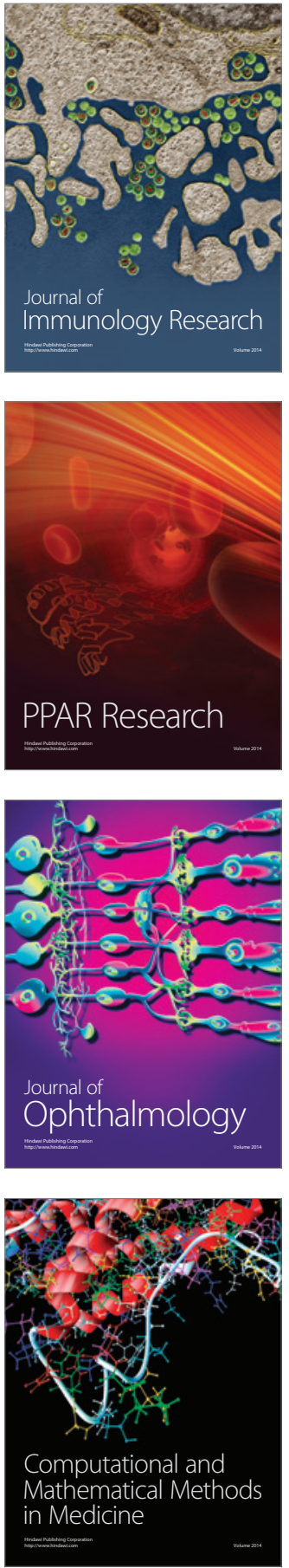

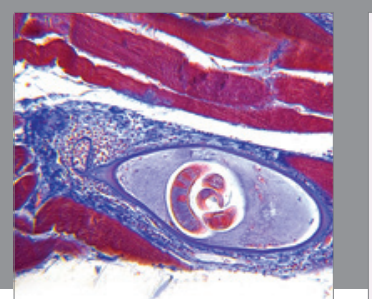

Gastroenterology Research and Practice

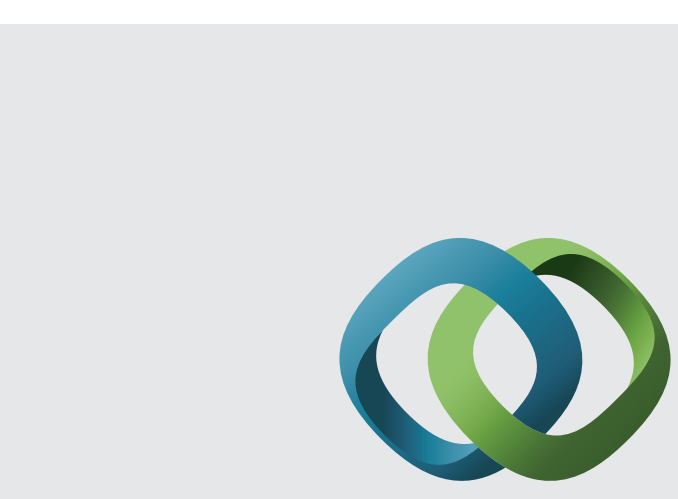

\section{Hindawi}

Submit your manuscripts at

http://www.hindawi.com
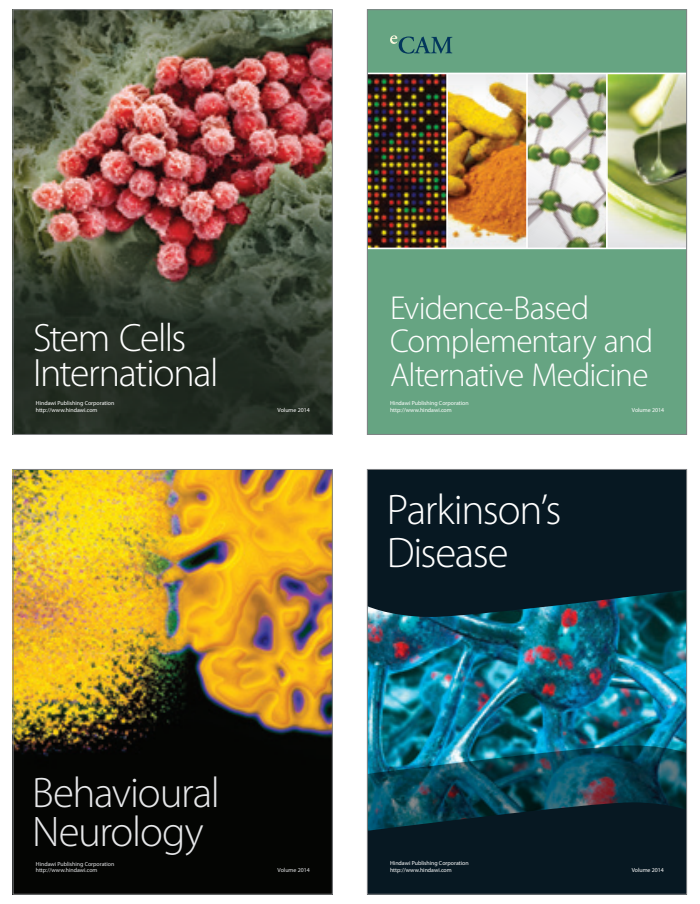
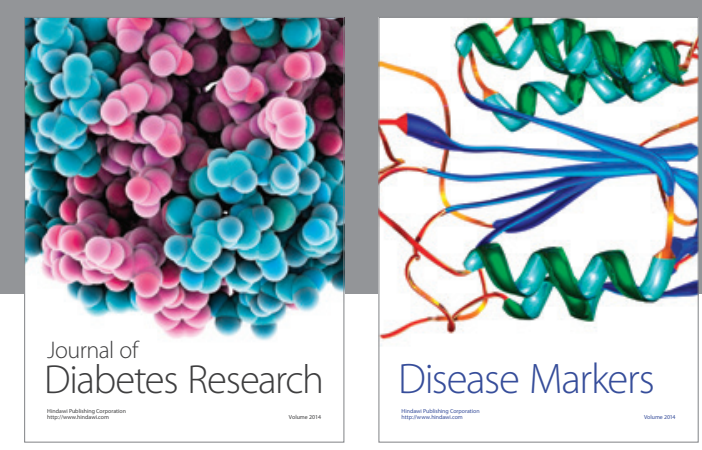

Disease Markers
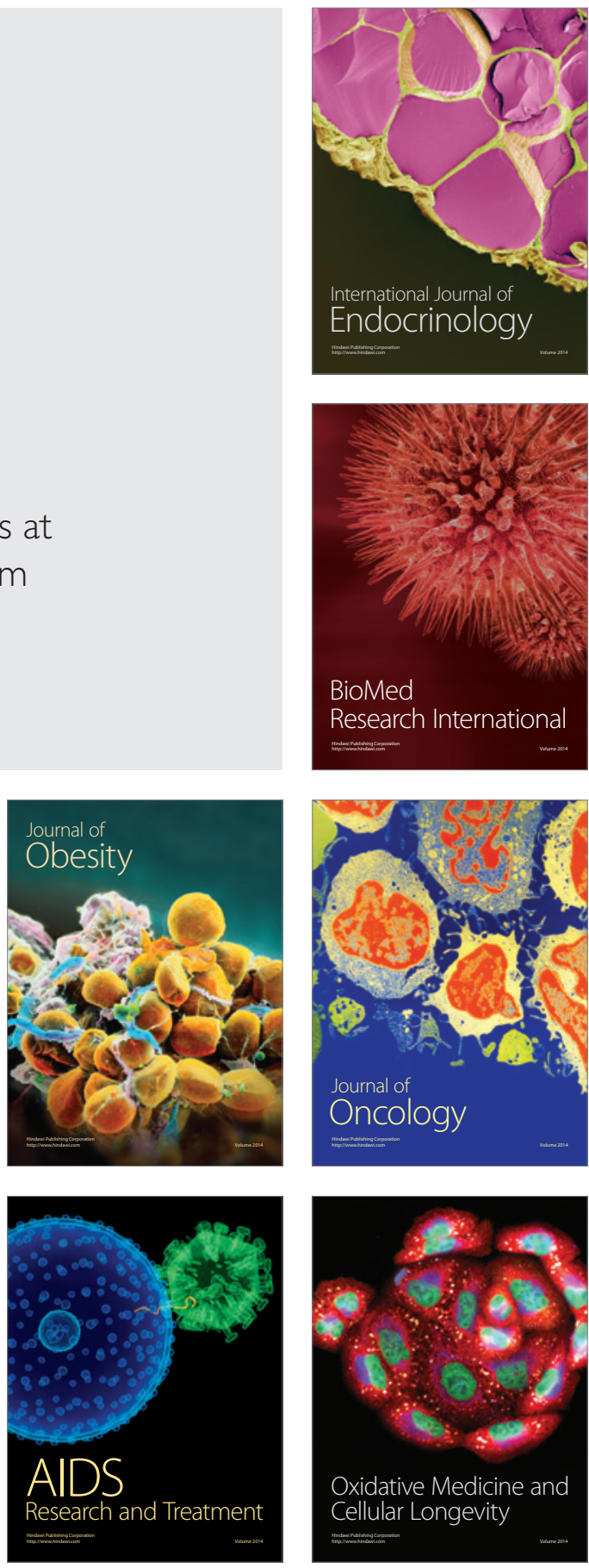